\title{
Intrinsic and extrinsic inhomogeneities in mixed-valence manganites
}

\author{
B. I. Belevtser* \\ B. Verkin Institute for Low Temperature Physics and Engineering, \\ National Academy of Sciences, pr. Lenina 47, Kharkov 61103, Ukraine
}

\begin{abstract}
It is suggested that extrinsic inhomogeneities in mixed-valence manganites deserve more attention and they should be taking into account on equal footing with hypothetical phase separation while examinating experimental data and developing the theoretical models of influence of stoichiometric and other types of inhomogeneities on properties of these and other transition-metal oxides.

PACS numbers: 72.80.Ga; 75.30.Vn; 64.75.+g
\end{abstract}

The structural, magnetic and electron transport properties of mixed-valence manganites of the type $\mathrm{R}_{1-x} \mathrm{~A}_{x} \mathrm{MnO}_{3}$ (where $\mathrm{R}$ is a rare-earth element, $\mathrm{A}$ a divalent alkaline-earth element) attracted much attention of scientific community in the last decade (see reviews 1, 2, 3, 4, 5, 6]). The interest is caused by observation of huge negative magnetoresistance (MR) near the Curie temperature, $T_{c}$, of the paramagneticferromagnetic transition for manganites with $0.2 \leq x \leq$ 0.5 . This phenomenon was called "colossal" magnetoresistance (CMR) and is expected to be used in advanced technology. The unique properties of mixed-valence manganites are determined by complex spin, charge and orbital ordered phases, and, therefore, are of great fundamental interest for physics of strongly correlated electrons. At present time, it is believed that one of the key feature of manganites is their intrinsic inhomogeneities in the form of coexisting competing ferromagnetic and antiferomagnetic/paramagnetic phases $[\underline{3}, 4,46]$. This phenomenon is generally called "phase separation". In Refs. [3, 6], theoretical computational models were developed for two cases: (1) Electronic phase separation which implies nanocluster coexistence. (2) Disorder-driven phase separation which leads to rather large (micrometer size) coexisting clusters. Existence of the nanoscale as well as micrometer size inhomogeneities in manganites were corroborated experimentally (see Refs. [3, [4, [6] and references therein). Some other examples of the phaseseparation models can be found in Refs. [4, 7, 8, 9, 10] (actually, there is a vast literature on the subject, but it can not be cited more fully in this short communication). On the basis of this, it is hoped to explain transport and magnetoresistive properties (including CMR) of manganites taking into account the phase separation effects.

In spite of enormous theoretical and experimental activity in the area of the phase separation in manganites, many questions (sometimes rather simple and naive) remain open. Intrinsic inhomogeneities are believed to arise for thermodynamical reasons so that relative fraction of competing phases should depend on temperature, pressure, and magnetic field. The known experimental

*belevtsev@ilt.kharkov.ua studies give numerous (predominantly indirect though) evidences of structural and magnetic inhomogeneities in manganites, but are they in all cases intrinsic? The point is that in all manganites the extrinsic inhomogeneities are inevitably present (even in single crystal samples). Extrinsic inhomogeneities arise due to various technological factors in the sample preparation. They can cause chemical-composition inhomogeneity (first of all in the oxygen content), structural inhomogeneities (polycrystalline or even granular structure), strain inhomogeneities and so on. It is easy to find in literature a lot of experimental studies in which finding of phase separation effects is proclaimed, but the interpretations are often doubtful. In such cases the effects of technological inhomogeneities are quite obvious or, at least, can not be excluded. In some cases the magnetic inhomogeneities, induced by extrinsic reasons, can depend significantly on temperature, pressure and magnetic field as well, and their apparent influence on magnetic and transport properties of mixed-valence manganites may agree generally with that of predicted by some of the numerous phase-separation theoretical models. It should be noted, however, that a quantitative comparison of the known models with experiment is practically impossible (or is too ambiguous).

Consider shortly the main sources of extrinsic inhomogeneities. Mixed-valence manganites are complex perovskite-like oxides consisting of, at least, four elements. Their properties are very sensitive to crystal imperfections, especially to the structural, composition and other types of inhomogeneity in crystal lattice. The crystal perfection (and corresponding level of inhomogeneity) depends strongly on method of preparation, and on preparation conditions for the given method. In rough outline, the following methods of manganite growth are used: 1) thin film growth (mostly with pulsed-laser deposition method); 2) solid-state reaction method; 3) floating zone method.

Thin manganite films can be prepared highly oriented or even single-crystal epitaxial with fairly perfect crystal lattice. The highest values of magnetoresistance were observed in thin films. But it should be taken into account that films are always in an inhomogeneous strained state due to inevitable substrate-film lattice interaction, that induces, as a rule, a considerable magnetic and magne- 
toresistance anisotropy [11]. Due to strained state, some other film properties (among other things, the value of $T_{c}$ ) can be quite different from these of bulk materials.

Consider some examples of extrinsic inhomogeneities in films. A comprehensive and thorough study (with high-resolution electron microscopy) 12] of epitaxial $\mathrm{La}_{1-x} \mathrm{Ca}_{x} \mathrm{MnO}_{3}(x \approx 0.3-0.35)$ films grown on $\mathrm{SrTiO}_{3}$ substrate has revealed that close to substrate a perfectly coherent strained layer is formed, above which crystal blocks with columnar structure grow; these blocks and boundary regions between them accomodate the lattice mismatch between substrate and film. Boundary regions between the blocks (domains) are non-stoichiometric, having deficiency of oxygen and of lanthanum. Similar results are found in Ref. [13, where secondary-phase nonstoichiometric rods were found in $\mathrm{La}_{0.7} \mathrm{Ca}_{0.3} \mathrm{MnO}_{3}$ films grown on $\mathrm{LaAlO}_{3}$ and $\mathrm{SrTiO}_{3}$ substrates. The films have a domain structure, in which the rods are believed to be responsible for relieving stress during film growth. Magnetic force microscopy study of pulsed laser deposited $\mathrm{La}_{1-x} \mathrm{Sr}_{x} \mathrm{MnO}_{3}(x=0.23$ and 0.3$)$ films [14] have revealed local FM regions at temperature above the $T_{c}$ of the film. These regions with higher $T_{c}$ were found around the grain boundaries and attributed to the local variation of strain in film. The above examples show that even epitaxial films, prepared at optimal conditions, have inhomogeneous strains and a local non-stoichiometry, that can play a significant role in transport and magnetoresistive properties of thin films.

The solid-state reaction (SSR) technique enables preparing ceramic or polycrystalline samples. The crystal quality (and, therefore, resistive, magnetoresistive and magnetic properties) of the SSR samples depends in crucial way on preparation conditions, especially on sintering and annealing temperature. In samples prepared with optimal sintering temperature, a fairly sharp resistive and magnetic transitions near $T_{c}$ are observed; whereas, quite different resistive and magnetization behavior is seen for samples with the same nominal composition, but prepared at low temperature [15]. This is to be attributed to composition and structure inhomogeneity of samples sintered at low temperatures. In all preparation conditions, however, SSR samples are always polycrystalline and contain inevitably at least one source of inhomogeneity: grain boundaries regions. These are regions of structural, magnetic and stoichiometric disorder, and, therefore, they have different conducting and magnetic properties as compared with these inside the grains. Beside this, a rather appreciable composition inhomogeneities (not associated with grain boundaries) can not be excluded in SSR samples even when they are prepared at optimal conditions. The common methods of checking of stoichiometric inhomogeneity and mixed-phase state (x-ray powder diffraction or electron microprobe analysis) have too low accuracy to come to unambiguous conclusion about composition homogeneity. For example, if a sample is a mixture of two phases of $\mathrm{R}_{1-x} \mathrm{~A}_{x} \mathrm{MnO}_{3}$, composed from the same elements, but with appreciably different values of $x$ or oxygen concentration, it is hard or even impossible to see clearly enough the two-phase state in diffraction pattern, even if volume fractions of the phases are comparable; whereas, magnetic and other properties of these phases can be significantly different. Only non-perovskite-type impurities can be detected quite clearly down to $2 \%$. Electronic microprobe elemental analysis has an accuracy about $\pm 5 \%$, in most cases, leaving room for stoichiometic disorder within these limits. More powerful, but much more expensive methods, like neutron diffraction or small-angle neutron scattering, are not in common use, but even these methods have their limits of accuracy. Since properties of manganites are very sensitive to chemical composition and, therefore, to stoichiometric disorder, no wonder to find in literature quite different properties of manganites of the same nominal composition, prepared by SSR method. In spite of the unavoidable technological inhomogeneity, the SSR method is in common use for preparation of mixedvalence manganites of various composition. The reason is that SSR method appears to be not very sophisticated (at least, at first glance) and does not require an expensive equipment. At proper experience and rather hard work, it is possible to obtain polycrystalline samples of rather good quality with sharp resistive and magnetic transitions. For example, a generally recognized phase diagram for system $\mathrm{La}_{1-x} \mathrm{Ca}_{x} \mathrm{MnO}_{3}$ was obtained for SSR polycrystalline specimens [5].

It is easy to find in literature hundreds of papers devoted to the film or bulk ceramic manganites, but far less studies concern single-crystal samples. The obvious reason is that it is not so easy to prepare manganite single crystals. But even single-crystals prepared by the floating zone method are not free from defects and extrinsic inhomogeneities. Really, they have mosaic blocks, twins, inhomogeneous strains, and stoichiometric disorder [16, 17, 18].

The experimental data provide, therefore, that the technological inhomogeneities are unavoidable for any preparation method, and actually they can be called "intrinsic" as well. For this reason, (i) in many cases it is better to speak about multiphase coexistence instead of the phase separation; (2) the technological inhomogeneities should be directly taken into account in new theoretical models. The latter demand is conditioned by the circumstance that manganite materials which can be used in an advanced technology will surely have some crystal imperfections or inhomogeneities. Moreover, in some cases specific types of inhomogeneities should be even induced specially to provide necessary properties. For example, grain boundaries or specially prepared percolation structures can ensure high MR in low fields in temperature range far below $T_{c}$, that may be necessary for some applications.

As for the phase separation, this concept becomes now, on the one hand, a commonplace, but, on the other hand, the term is too general to imply something specific. At interpretation of their results, experimentalists often speak 
quite generally about phase separation or just mention it, meaning not much at that. And how they can, if at least a dozen of diverse models (suggesting quite different mechanisms of phase separation) are developed, which, however, practically can not be numerically compared with experiment? In spite of this, the phase-separation concept appears to be very attractive since it can give a quite natural qualitative explanation for both the huge drop in resistance and the CMR in vicinity of magnetic transitions in manganites, taking into account a percolating character of these transitions [4, 8]. Consider, for example, $\mathrm{La}_{1-x} \mathrm{Ca}_{x} \mathrm{MnO}_{3}$ system. According to Refs. 19, 20, 21, 22, 23, 24], paramagnetic-ferromagnetic (PMFM) transition in this compound is of first order for the range $0.25<x<0.4$. It is found in these compounds that FM metallic clusters are present well above $T_{c}$, while some PM insulating clusters can persist down to a range far below $T_{c}$ 25, 26, 27]. That seems naturally for first-order transition where nucleation of the FM clusters above $T_{c}$ is quite expected, as well as the presence of some amount of PM clusters below $T_{c}$. After all, transition of this type is hysteretic and depends on the rate of heating or cooling. In this case a real phase separation and percolation processes can be expected around $T_{c}$. Since the PM phase is insulating and the FM one is metallic, some kind of insulator-metal transition takes place near $T_{c}$. The technological inhomogeneities broaden the temperature range of the PM-FM transition so that it may appear more smooth and continuous, like second order transition.

For Ca concentration outside of the above-indicated range, $0.25<x<0.4$, the PM-FM transition is found to be of second order in $\mathrm{La}_{1}{ }_{x} \mathrm{Ca}_{x} \mathrm{MnO}_{3}$ samples with $x=0.20,0.40$ and $0.45[22,29,30]$. According to the phase diagram for this system $[1,3,[3,[6]$, these concentrations are close to critical ones: $x \approx 0.2$ (which is a border between the FM metallic and insulating states) and $x=0.5$ (which is a border between FM metallic and insulating charge-ordered states). It is clear that unavoidable technological stoichiometric disorder will have a greater impact on magnetic transition for samples having nominal $\mathrm{Ca}$ concentrations near the above-mentioned critical values. The $T_{c}$ value depends rather strongly on $x$ near these threshold concentrations; whereas, the concentration dependence of $T_{c}$ near the optimal doping $(x \approx 0.35)$ is rather weak (see the phase diagram in Refs. [1, 3, 5, 6]). In this case, the magnetic transition for a sample with non-optimal concentration should be broader, than that for the optimal-doped samples, even if the level of composition-inhomogeneity is equal in both cases. It can not be excluded, therefore, that a second order transition found for these $\mathrm{La}_{1-x} \mathrm{Ca}_{x} \mathrm{MnO}_{3}$ samples is just rather broadened (smeared) first order transition.

It should be noted that PM-FM transition is found to be of second order in Sr-doped $\mathrm{La}_{1-x} \mathrm{Sr}_{x} \mathrm{MnO}_{3}$ samples $(x=0.3$ and 0.33$)$ as well [19, 31]. The Sr manganites are more conductive than Ca manganites and have much higher $T_{c}$ (maximum $T_{c}$ are about $260 \mathrm{~K}$ and $370 \mathrm{~K}$ for
$\mathrm{Ca}$ and Sr manganites, respectively). It seems that manganites with higher conductivity and $T_{c}$ are more prone to second order transition than those with low conductivity and $T_{c}$. In homogeneous samples with perfect crystal lattice the second order transition from PM to FM state should proceed at once in all sample volume as soon as the temperature cross $T_{c}$ going from above. No nuclei of FM phase above $T_{c}$, no supercooling or hysteresis phenomena should occur at this transition. Only thermodynamical fluctuations of the order parameter (the magnetization) are expected, which, however, should be confined to narrow critical region around $T_{c}$ 32, 33. These fluctuations of magnetic order have usually a rather noticeable effect on "non-magnetic" properties, like temperature coefficient of the resistivity, heat capacity, magnetoresistance, thermal expansion, in vicinity of $T_{c}$ 32, 33].

Stoichiometric disorder and non-homogeneous strains of crystal lattice, which are unavoidable in real manganites due to the above-indicated technological reasons, can undoubtedly have a pronounced effect on the secondorder PM-FM transitions. This effect has long been known and considered for simple FM metals [33]. Take, for example, as in Ref. [33], a system consisting of multiple phases with different $T_{c}$. There is some volume distribution of regions with different $T_{c}$ within the sample. Availability of interphase transition regions between different phases should be taken into account as well. The temperature dependence of the magnetization for this sample will show somewhat broaden PM-FM transition 33] (the temperature width of the transition depends on how wide is the distribution of $T_{c}$ in the sample). From that an averaged $T_{c}$ value can be determined. But some parts of the sample have $T_{c}$ greater or less than this averaged value. Therefore, it can be found with some experimental methods that some FM clusters exist above $T_{c}$, with their volume increasing when going to $T_{c}$ from above; whereas, PM clusters can be found below $T_{c}$, with their volume fraction decreasing when going down away from $T_{c}$. The reason for this behavior is quite obvious taking into account the sample inhomogeneity. Now, even if every single phase of this multiphase system undergoes a second order transition, the total character of transition will not that for homogeneous system. It will be of percolative nature. If PM and FM phase states differ drastically in their conductivity, the CMR can be found. Imagine that size of inhomogeneities is rather small, say, a few nanometers (which is quite possible for technological inhomogeneities). Is it possible in this case to attribute the magnetotransport behavior of this system near the PM-FM transition to the phase separation effect with some certainty? The negative answer is obvious since technological inhomogeneities alone can provide this behavior.

Due to enormous theoretical activity in this area, it is rather appropriate to believe that phase separation really takes place in manganites and in other transition-metal oxides (although it is difficult to make a right choice from numerous propositions of the phase separation mecha- 
nisms). But how to distinguish surely enough these thermodynamic effects from those of extrinsic inhomogeneity? It is a really difficult problem. I think that theoreticians should not disregard the influence of extrinsic inhomogeneities, but, on the contrary, they should take them into account in their models quite directly along with intrinsic inhomogeneities. This necessity was indicated quite clearly in the paper of Yukalov [10]. One of the principal ideas of this paper is that real systems are never free from external perturbations, that makes the system stochastically unstable even if external perturbations are infinitesimally small. After all, extrinsic inhomogeneities can even stimulate appearance of thermodynamic phase separation, so that some kind of interaction between them is possible.

In conclusion, at consideration of experimental data for mixed-valence manganites and developing of theoret- ical models for them, the unavoidable influence of extrinsic disorder and inhomogeneities should always be taken into account. These inhomogeneities can act separately as well as together with the suggested intrinsic inhomogeneities (phase separation) and determine to a great extent the magnetic and magnetotransport properties of these compounds. Although, for the most part, the known properties of $\mathrm{La}_{1-x} \mathrm{Ca}_{x} \mathrm{MnO}_{3}$ system near the PM-FM transition were used here for backing of the above-mentioned point of view, the general conclusion of this paper is applicable (in author opinion) to other magnetic transitions in manganites (for example, for transitions to charge-ordered states) and to related magnetic transition-metal oxides, like cobaltites $\mathrm{La}_{1-x} \mathrm{Sr}_{x} \mathrm{CoO}_{3}$.

Author sincerely acknowledges a very useful discussion of some questions, touched in this note, with Dr. P. A. Joy from National Chemical Laboratory, Pune, India.
[1] A. P. Ramirez, J. Phys.: Condens Matter 9, 8171 (1997).

[2] M. D. Coey, M. Viret, and S. von Molnar, Adv. Phys. 48, 167 (1999).

[3] E. Dagotto, T. Hotta, and A. Moreo, Phys. Rep. 344, 1 (2001).

[4] E. L. Nagaev, Phys. Uspekhi 39, 781 (1996); Phys. Rep. 346, 387 (2001).

[5] K.H. Kim, M. Uehara, V. Kiryukhin, and S-W. Cheong, in Colossal magnetoresistive manganites, T. Chatterji (ed.), Kluwer Academic Publ., Dordrecht, Netherlands (2003); preprint cond-mat/0212113

[6] E. Dagotto, J. Burgy, and A. Moreo, Solid State Commun. 126, 9 (2003); E. Dagotto, preprint cond-mat/0302550

[7] N. D. Mathur, P. B. Littlewood, Solid State Commun. 119, 271 (2001).

[8] A. O. Sboychakov, A. L. Rakhmanov, K. I. Kugel, M. Yu. Kagan, and I. V. Brodsky, JETP 95, 753 (2002).

[9] G. Iadonisi, V. Cataudella, G. De Fillipis, V. Marigliano Ramaglia, C. A. Perroni, and F. Ventriglia, Phys. Stat. Sol. B 237, 215 (2003).

[10] V. I. Yukalov, preprint cond-mat/0308127

[11] B. I. Belevtsev, V. B. Krasovitsky, D. G. Naugle, K. D. D. Rathnayaka, A. Parasiris, S. R. Surthi, R. K. Pandey, and M. A. Rom, Phys. Stat. Sol. A 188, 1187 (2001)

[12] O. I. Lebedev, G. Van Tendeloo, A. M. Abakumov, S. Amelinckx, B. Leibold, and H.-U. Habermeier, Phil. Mag. A 79, 1461 (1999); G. Van Tendeloo, O. I. Lebedev, and S. Amelinckx, J. Magn. Magn. Mater. 211, 73 (2000).

[13] C. J. Lu, Z. L. Wang, C. Kwon, and Q. X. Jia, J. Appl. Phys. 88, 4032 (2000).

[14] Yeong-Ah Soh, G. Aeppli, N. D. Mathur, and M. G. Blamire, J. Appl. Phys. 87, 6743 (2000).

[15] P. A. Joy, P. S. Anil Kumar, and S. K. Date, Mater. Res. Bull. 14/15, 2143 (1999).

[16] D. Shulyatev, S. Karabashev, A. Arsenov, Ya. Mukovskii, and S. Zverkov, in Defects and Surface-Induced Effects in Advanced Perovskites, G. Borstel et al. (eds.), Kluwer Academic Publishers, Netherlands (2000), pp.455-460.

[17] D. Shulyatev, S. Karabashev, A. Arsenov, Ya. Mukovskii, and S. Zverkov, J. Cryst. Growth 237-239, 810 (2002).
[18] Bas B. Van Aken, A. Meetsma, Y. Tomioka, Y. Tokura, and Thomas T. M. Palstra, Phys. Rev. B 66, 224414 (2002); B.B. Van Aken, Ph.D. Thesis, University of Groningen (2001), www.ub.rug.nl/eldoc/dis/science.

[19] J. Mira and J. Rivas, Phys. Rev. B 60, 2998 (1999).

[20] H. S. Shin, J. E. Lee, Y. S. Nam, H. L. Ju, and C. W. Park, Solid State Commun. 118, 377 (2001).

[21] Y. B. Zhang, S. Li, P. Hing, C. Q. Sun, W. Gao, and S. X. Dou, Solid State Commun. 120, 107 (2001).

[22] Chang Seop Hong, Wan Seop Kim, and Nam Hwi Hur, Phys. Rev. B 63, 092504 (2001).

[23] J. E. Gordon, C. Marcenat, J. P. Franck, I. Isaac, Guanwen Zhang, R. Lortz, C. Meingast, F. Bouquet, R. A. Fisher, and N. E. Phillips, Phys. Rev. B 65, 024441 (2002).

[24] C. P. Adams, J. W. Lynn, V. N. Smolyaninova, A. Biswas, R. L. Greene, W. Ratcliff II, S-W. Cheong, Y. M. Mukovskii, and D. A. Shulyatev, preprint cond-mat/0304031

[25] J. M. De Teresa, M. R. Ibarra, P. A. Algarabel, C. Ritter, C. Marquina, J. Blasco, J. Garcia, A. del Moral, and Z. Arnold, Nature 386, 256 (1997).

[26] Joonghoe Dho, Ilryong Kim, Soonchil Lee, K. H. Kim, H. J. Lee, J. H. Jung, and T. W. Noh, Phys. Rev. B 59, 492 (1999).

[27] G. Papavassiliou, M. Fardis, M. Belesi, T. G. Maris, G. Kallias, M. Pissas, D. Niarchos, C. Dimitropoulos, and J. Dolinsek, Phys. Rev. Lett. 84, 761 (2000).

[28] X. Bohigas, J. Tejada, M. L. Marínez-Sarrión, S. Tripp, and R. Black, J. Magn. Magn. Mater. 208, 85 (2000).

[29] J. J. Rhyne, H. Kaiser, L. Stumpe, J. F. Mitchell, T. McCloskey, and A. R. Chourasia, J. Magn. Magn. Mater. 226, 775 (2001).

[30] D. Kim, B. Revaz, B. L. Zink, F. Hellman, J. J. Rhyne, and J. F. Mitchell, Phys. Rev. Lett. 89, 227202 (2002).

[31] P. Novák, M. Maryŝko, M. M. Savosta, and A. N. Ulyanov, Phys. Rev. B 60, 6655 (1999).

[32] S. V. Vonsovsky, Magnetizm, Nauka, Moscow (1971).

[33] K. P. Belov, Magnitnie prevrashcheniya (Magnetic transitions), Gos. Izdat. Fiz.-Mat. Lit., Moscow (1959). 